\title{
Fixed interval behavior in mixed and multiple schedules with alternating fixed interal-fixed ratio components
}

\author{
Stephen B. Kendall \\ UNIVERSITY OF ALABAMA
}

\begin{abstract}
Abstraet
Two pigeons were run on both multiple and mixed FI-1 FR-10 schedules with the schedule components occurring in a simple alternating order. Data for the FI components showed that FI performance was quantitatively similar for both multiple and mixed schedules. Observation of the subjects revealed that they adopted unusual response topographies in the FR component of the mixed schedule.
\end{abstract}

\section{Introduetion}

Ferster and Skinner (1957) report that after training on a multiple FI-FR schedule with the schedule components in simple alternation, rats switched to a mixed schedule, with the same schedule components in the same sequence, rapidly begin to perform in a fashion which is indistinguishable from performance in the multiple schedule. In the multiple schedule exteroceptive discriminative stimuli are available upon which the subject can base a discrimination. If these stimuli are absent, as they are in a mixed schedule, and if the subjects still perform as if they were on the multiple schedule, the relevant discriminative stimuli must arise from the subject's own behavior.
The present experiment explores this phenomenon with pigeons and attempts to compare the shape of the fixed interval scallops in multiple and mixed schedules where the components occur in a simple alternating order.

\section{Method}

Subjects were two experimentally sophisticated homing pigeons, one male and one female. Their ages are unknown except that they were not over 3-yr. old at the time of the experiment. They were run at $70 \%$ of freefeeding weight.

The experimental chamber consisted of a two-key pigeon panel housed in a 3/4-in plywood box. Only one key was used in this experiment. A motor driven grain hopper was used in place of the standard solonoid operated feeder. Standard relay and timing devices were used to program the contingencies. Responses were recorded on a cumulative recorder and a bank of four counters. The pigeon chamber and control apparatus were housed in separate rooms.

In the first step of the training procedure the birds were run on a multiple FI-1 (min.) FR-2 schedule. After this training they were run on a mixed schedule with the same components. During this training the

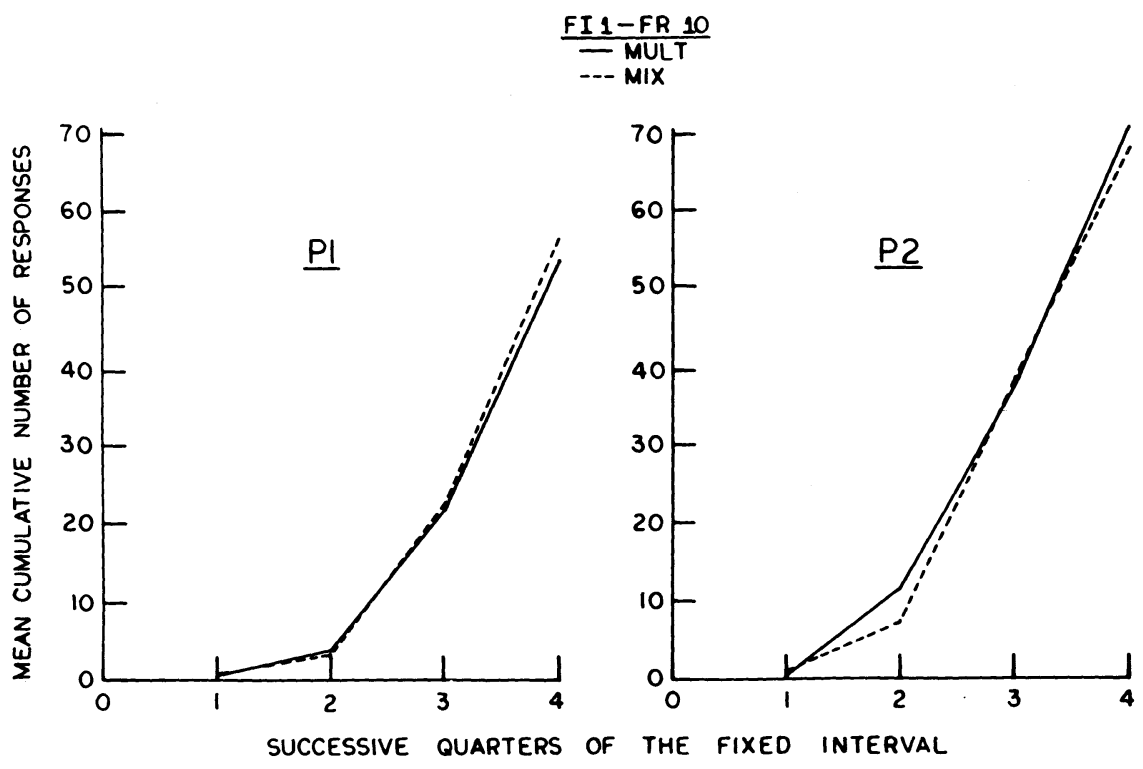

Fig. 1. Mean cumulative number of responses in successive quarters of the fixed interval for mixed (dashed) and multiple (solid) schedules. Data are plotted separately for the two subjects. 
schedule components always appeared in simple alternation; each FI was followed by an FR and each FR by an FI. When the birds had demonstrated reasonable proficiency at these schedules they were shifted to multiple FI 1 FR 10. After stability had been achieved on this schedule they were again shifted to mixed FI 1 FR 10. During all multiple schedules a blue light illuminated the key during the FR component. No key light was on during the FI component. During the mixed schedules no key lights were on in either component. As before, the components appeared in simple alternation. The birds were given 52 reinforcements per session.

\section{Results}

Since the FI-1 FR-2 schedules were run as training procedures, no data from that situation are presented here. Figure 1 shows the FI performance on the multiple and mixed schedules for each bird separately. These data were collected by dividing the FI-1 component into four 15-sec. periods. All responses occurring in the first $15 \mathrm{sec}$. after reinforcement were totaled on the first counter; all responses in the next 15-sec. period were totaled on the second counter and so on. Each point represents the mean of the last four days of data collection on that schedule. The curves are cumulative so that the last point represents the average total number of responses for the fixed interval components. The solid line is the multiple schedule data and the dashed line is the mixed schedule data.

The curves for P1 are quite similar. The widest separation between the two being at the last point showing a very slight tendency for more responses in the mixed schedule. Although the curves for P2 show some differences, especially in the second quarter of the interval, the difference is in the opposite direction from what might be expected if stimulus control over the FI component were poorer in the mixed schedule.

The similarity of the mixed and multiple schedule curves for each bird can be taken to mean that the FI performances were quantitatively similar under mixed and multiple schedule conditions.

No differences in FR behavior between mixed and multiple schedules could be detected by inspection of the cumulative records.

\section{Diseussion}

The results of this experiment indicate no lack of stimulus control over FI behavior in a mixed FI-FR schedule where the schedule components are in simple alternation. This result is in agreement with Ferster and Skinner's (1957) data from a similar experiment with rats. Proprioceptive stimulation arising from the FR component of the schedule is as effective in controlling the FI behavior as the change in exteroceptive stimulation which occurs in the multiple schedule.

Observation of the subjects suggests that they mediate performance in the mixed schedule by adopting different response topographies in the two different schedule components. While different topographies in the FI and FR component of the multiple schedule were observed they were not nearly so dramatic as those observed in the mixed schedule. P1 adopted the technique of pecking at the key with his head nearly upside down during the FR component of the mixed schedule. His pecking during the FI component was done with his head rightside up, in the normal fashion. P2 stood to the side of the key and struck it from an angle during the FR component of the mixed schedule and behaved similarly to P1 during the FI component.

The control of the FR behavior in the mixed schedule must arise in the same way as control of the FI behavior; that is, it is controlled by the FI behavior. No FR data are presented, however, since any expected differences between the mixed and multiple schedules should lie in the FI component. Ferster and Skinner (1957) have shown that in a mixed FI-FR schedule where the components occur in an irregular sequence the subjects perform in a manner appropriate to the ratio schedule after every reinforcement. If no reinforcement is forthcoming the subject then begins to behave in a manner appropriate to the interval schedule. This effect was observed in the present experiment during the transition from the multiple to the mixed schedule.

\section{Reference}

FERSTER, C. B., \& SKINNER, B. F. Schedules of reinforcement. New York: Appleton-Century-Crofts, 1957. 